\title{
Purified Adult Ensheathing Glia Fail to Myelinate Axons under Culture Conditions that Enable Schwann Cells to Form Myelin
}

\author{
Giles W. Plant, ${ }^{1}$ Paul F. Currier, ${ }^{1}$ Ernesto P. Cuervo, ${ }^{1}$ Margaret L. Bates, ${ }^{1}$ Yelena Pressman, ${ }^{1}$ \\ Mary Bartlett Bunge, ${ }^{2,3}$ and Patrick M. Wood ${ }^{2}$ \\ 1 The Chambers Family Electron Microscopy Laboratory, The Miami Project To Cure Paralysis, and Departments of \\ ${ }^{2}$ Neurological Surgery and ${ }^{3}$ Cell Biology and Anatomy, University of Miami School of Medicine, Miami, Florida 33136
}

Several studies have suggested that olfactory ensheathing glia (EG) can form Schwann cell (SC)-like myelin. Because of possible misinterpretation attributable to contaminating SCs, the capacity of EG to produce myelin needs to be explored further. Therefore, we compared the abilities of adult EG, purified by immunopanning with p75 antibody, and adult SCs to produce myelin when cocultured with purified dorsal root ganglion neurons (DRGNs) in serum-free and serum-containing media. In both media formulations, the number of myelin sheaths in SC/DRGN cultures was far higher than in EG/DRGN cultures; the number of sheaths in EG/DRGN cultures was equal to that in purified DRGN cultures without added cells. The latter result demonstrates that myelination by a few SCs remaining in purified DRGN cultures may occur, suggesting that myelin in EG/DRGN cultures could be SC myelin. Striking differences in the relationship of EG and SC processes to axons were observed. Whereas SCs displayed relatively short, thick processes that engulfed axons in small bundles or in individual cytoplasmic furrows and segregated larger axons into one-toone relationships, EG extended flattened sheets that partitioned or only partially encircled fascicles of axons, sometimes spanning the entire culture. SCs exhibited behavior typical of SCs in peripheral nerves, whereas EG exhibited characteristics resembling those of $E G$ in olfactory nerves. In sum, p75selected EG from adult animals did not exhibit an SC-like relationship to axons and did not form myelin.

Key words: neuron-glia coculture; Schwann cells; olfactory bulb; olfactory ensheathing glia; myelination; ascorbate; glial plasticity
Ensheathing glia (EG) are found in olfactory mucosa, nerves, and bulbs where axonal growth occurs throughout life. Axonal regeneration is fostered by transplanting suspensions of olfactory bulb cells (containing EG) or purified EG (Ramón-Cueto and NietoSampedro, 1994; Li et al., 1997, 1998; Ramón-Cueto et al., 1998, 2000). Therefore, EG transplantation has been proposed as an alternative to Schwann cell (SC) transplantation in CNS repair. Whereas an SC bridge promotes regrowth of axons across an area of injury in spinal cord, the fibers do not leave the bridge to enter the distal cord (Xu et al., 1997; for review, see Plant et al., 2001). The SCs, unlike EG, do not migrate into astrocyte-containing areas (for review, see Franklin and Barnett, 1997).

However, SCs present important advantages nonetheless. Both rat and human SCs can be generated in large numbers. After growth in culture, 50 billion SCs can be obtained from one adult human sural nerve (Casella et al., 1996; P. Wood, unpublished observations), enough to construct a graft of $1 \mathrm{~cm}^{2}$ cross-sectional area and $4 \mathrm{~m}$ in length. Thus, autologous SC grafts can be

\footnotetext{
Received Nov. 13, 2001; revised May 7, 2002; accepted May 7, 2002.

This research was supported by the Christopher Reeve Paralysis Foundation, the International Spinal Research Trust, National Institutes of Health Grant NS09923, and the Miami Project to Cure Paralysis. We thank Jennifer L. Katz and Francisco J. Cruz-McRea for excellent technical assistance and Diana Masella for word processing. The gift of the 192 hybridoma cell line from Dr. Eric Shooter (Stanford University, Stanford, CA) is gratefully acknowledged.

Correspondence should be addressed to Dr. Patrick Wood, The Miami Project to Cure Paralysis, The University of Miami Medical School, P.O. Box 016960, Mail Locator R-48, Miami, FL 33101. E-mail: pwood@miami.edu.

G. W. Plant's present address: Red's Spinal Cord Research Laboratory, School of Anatomy and Human Biology (CTEC) and WAIMR, 35 Stirling Highway, Crawley, Perth WA 6009, Australia.

Copyright (C) 2002 Society for Neuroscience $\quad 0270-6474 / 02 / 226083-09 \$ 15.00 / 0$
}

prepared. Moreover, SCs reliably provide myelin for axons in the CNS environment.

Do EG form myelin as well? In the olfactory system they do not; they surround bundles of nonmyelinated axons. When adult rat olfactory bulb EG were cultured with olfactory epithelium, the EG enfolded but did not myelinate small diameter neurites (Ramón-Cueto et al., 1993). However, Devon and Doucette (1992) found myelin in cocultures of unpurified fetal olfactory bulb EG and dorsal root ganglion neurons (DRGNs). The myelinating cells closely resembled SCs, including their envelopment by basal lamina. Moreover, EG, unlike SCs, formed myelin in ascorbate-low medium (Devon and Doucette, 1995).

Imaizumi et al. (1998) transplanted cell suspensions from neonatal olfactory bulb nerve layers into adult rat dorsal columns demyelinated by $\mathrm{x}$-irradiation and ethidium bromide injections. SC-type remyelination was extensive and conduction properties were improved. Using the same model, Franklin et al. (1996) transplanted suspensions of a clonal olfactory bulb-EG cell line to ensure the introduction of purified populations of cells (although additional cell types were found later); again, demyelinated fibers became myelinated. Myelination was also seen after grafting of suspensions of adult human olfactory bulb cells (Kato et al., 2000) or purified adult human EG (Barnett et al., 2000) into areas of persistent demyelination in rat spinal cord, or after grafting of olfactory bulb cells into lesioned corticospinal tract ( $\mathrm{Li}$ et al., 1997, 1998). The myelinating cells were morphologically indistinguishable from SCs. In studies of this type it is important to rule out the possibility that the transplanted EG might induce or enhance the migration of endogenous SCs into the transplant site (Bunge et al., 1994; Brook et al., 1998). 
In the present study, adult rat olfactory bulb cells were immunopanned with p75 antibody to enrich for EG. Then myelination in EG/DRGN cocultures was compared with that in SC/DRGN cocultures under identical conditions. Evidence for EG myelination was not obtained.

\section{MATERIALS AND METHODS}

SC cultures. SCs were obtained from adult female Fischer 344 rats. The method of isolation was described by Morrissey et al. (1991). Briefly, sciatic nerves from five rats were cut into small 1-2 $\mathrm{mm}$ pieces and placed into $35 \mathrm{~mm}$ uncoated tissue culture dishes. Every week nerve segments were transferred to new $35 \mathrm{~mm}$ dishes until, after 3 weeks in culture, the nerve segments (essentially depleted of fibroblasts) were enzymatically and mechanically dissociated before transferring to new dishes for SC expansion in the presence of mitogens $(20 \mu \mathrm{g} / \mathrm{ml}$ bovine pituitary extract and $2 \mu \mathrm{M}$ forskolin). SC purity was between 95 and $98 \%$.

$E G$ cultures. Primary EG cultures were prepared as described previously by Ramón-Cueto and Nieto-Sampedro (1992). Briefly, four 3- to 4-month-old adult female Fischer rats (Charles River Laboratories, Wilmington, MA) were killed by decapitation, and the olfactory bulbs were extirpated and placed in Leibovitz's L-15 medium (Invitrogen, Grand Island, NY). After carefully removing the pia, the olfactory nerve fiber layers were dissected from the rest of the bulb. Care was taken to minimize the inclusion of non-nerve fiber layer bulb tissue, but it is very likely that some glomerular-layer tissue was unintentionally included. The tissue fragments were cut into $1 \mathrm{~mm}^{3}$ pieces and incubated with $0.25 \%$ trypsin (Worthington Biochemical Corporation, Lakewood, NJ) and $50 \mu \mathrm{g} / \mathrm{ml}$ DNase (Sigma, St. Louis, MO) at $37^{\circ} \mathrm{C}$ for $60 \mathrm{~min}$ with continual shaking. Trypsinization was stopped by adding DMEM (Invitrogen) and Ham's F-12 medium (Invitrogen) (1:1 mixture) supplemented with $10 \%$ fetal bovine serum (DF-10S medium). The dissociated cells were plated on poly-L-lysine-coated tissue culture dishes in DF-10S medium supplemented with bovine pituitary extract $(20 \mu \mathrm{g} / \mathrm{ml}$; Invitrogen) and forskolin $(2 \mu \mathrm{M})$.

The method used to purify EG from primary cultures was modified from the original protocol (Ramón-Cueto and Nieto-Sampedro, 1992). After 6-7 d, EG were separated from other cell types in primary cultures by immunopanning, using an antibody against the p75 nerve growth factor receptor (gift from Dr. Eric Shooter, Stanford University, Stanford, CA). The culture period before immunopanning allowed the cells in the primary cultures to proliferate, thereby increasing cell yields and minimizing losses encountered when processing cells in low numbers. Cells from primary cultures were detached with $0.05 \%$ trypsin and $0.02 \%$ EDTA (Invitrogen), centrifuged, and washed two times with DF-10S. Cells were suspended in L-15 medium and plated on $100 \mathrm{~mm}$ Petri dishes that had been pretreated sequentially with antibodies as follows: antimouse IgG antibody (1:100) (Jackson ImmunoResearch, West Grove, PA) overnight at $4^{\circ} \mathrm{C}$, washing three times with L-15 medium, p75 antibody at a 1:5 dilution in L-15 medium with $5 \%$ fetal bovine serum for $2 \mathrm{hr}$ at $4^{\circ} \mathrm{C}$, and washing three times with L-15. Unpurified cells were plated onto the antibody-treated dishes at a density of $4 \times 10^{5}$ cells/dish for $30 \mathrm{~min}$ at $4^{\circ} \mathrm{C}$. To remove unbound cells, dishes were washed five times with L-15 medium. Bound cells were detached from dishes with a cell scraper (Caster, Cambridge, MA), centrifuged, and resuspended in DF-10S. Cells were seeded onto poly-L-lysine-treated (average molecular weight, 30,000; $200 \mu \mathrm{g} / \mathrm{ml}$; Sigma) $100 \mathrm{~mm}$ dishes and fed DF-10S containing pituitary extract and forskolin as noted above.

$D R G N$ cultures. Details of DRGN culture preparation have been described by Kleitman et al. (1998). In brief, DRGs dissected from embryonic day 15 Sprague Dawley rats were first incubated in trypsin (Worthington Biochemical Corporation, Freehold, NJ). Trypsin activity was stopped by the addition of L-15-containing fetal bovine serum. The sample was centrifuged and the pellet was resuspended in serumcontaining medium. The sample was triturated with a pipette until the neurons were dispersed, the volume was increased to $5 \mathrm{ml}$, the suspension was mixed and centrifuged, and the pellet was resuspended in NLAmedium (neurobasal medium plus B27, both from Invitrogen, supplemented with nerve growth factor at $\sim 10 \mathrm{ng} / \mathrm{ml}$, but without ascorbate) (1-1.5 ganglia per drop). The addition of nerve growth factor to the medium ensured the survival of DRGN for the duration of the experiment (Kleitman et al., 1998). NLA+ medium was the same as NLAmedium but with the addition of $50 \mu \mathrm{g} / \mathrm{ml}$ ascorbic acid. One drop of DRG cell suspension in NLA - medium was plated in the center of dry, ammoniated collagen-coated Aclar minidishes (Kleitman et al., 1998).
The drop of cell suspension remained where placed on the hydrophobic collagen surface if the cultures were protected from mechanical disturbance. Approximately 5000-7000 neurons were plated per culture. After the cells attached to the collagen overnight, the cultures were flooded and subsequently treated with NLA - medium containing fluorodeoxyuridine (FUDR) on days $2-4,6-8$, and $10-12$ to kill the non-neuronal cells (fibroblasts, SCs, and phagocytes). After this antimitotic treatment, the resulting DRGN cultures were maintained on NLA- medium for at least 1 week to ensure that no residual FUDR remained when SCs or EG were added.

Myelination in SC/DRGN and EG/DRGN cocultures. For myelination experiments in the absence of serum, cocultures were prepared by addition of $50,000 \mathrm{SCs}$ or $50,000 \mathrm{EG}$ to purified DRGN cultures. Two groups of DRGN cultures were kept without the addition of glial cells. The DRGN cultures and the cocultures were fed every $2-3 \mathrm{~d}$ with NLAmedium (without serum or ascorbate) for 2 weeks. For the induction of myelination, 12 cultures each of DRGN, SC/DRGN, and EG/DRGN were switched to medium containing ascorbic acid (i.e., NLA+ medium). In addition, 12 cultures each of DRGN, SC/DRGN, and EG/DRGN were continued in NLA- medium. All cultures were then maintained for an additional 2 weeks, with replacement of the appropriate medium every $2-3$ d. The total period of coculture was thus 4 weeks. Myelination was observed as expected in SC/DRGN cultures before the end of the coculture period.

In a second series of two experiments testing myelination in serumcontaining medium, DRGN, SC/DRGN, and EG/DRGN cocultures were prepared exactly as described above. Each experimental group contained six cultures. All cultures were maintained for 2 weeks in NLA - medium to allow the added glia to proliferate. To start myelination, all groups were switched to NLA+ medium containing $10 \%$ heatinactivated serum. To obtain myelination, after switching to this ascorbate-containing medium the cultures were maintained for 3 additional weeks (first experiment) or $10 \mathrm{~d}$ (second experiment). During these experiments, the cultures were re-fed every 2-3 d.

Immunocytochemistry. Cultures were stained with antibodies against p75, S100, neurofilament, and myelin basic protein (MBP). For p75 staining, live cultures were first rinsed three times with L-15 and incubated with a mouse monoclonal p75 supernatant (IgG-192; diluted 1:10 in $\mathrm{L}-15$ ) for $30 \mathrm{~min}$ at $4^{\circ} \mathrm{C}$. The cultures were rinsed three times with L-15 plus $10 \%$ heat-inactivated goat serum (HIGS) and then incubated with a goat anti-mouse tetramethylrhodamine-conjugated secondary antibody (1:100; ICN Cappel, Costa Mesa, CA) for $30 \mathrm{~min}$ at $4^{\circ} \mathrm{C}$. The secondary antibody was diluted in L-15 plus $10 \%$ HIGS. Cultures were rinsed three times with L-15 and fixed for $15 \mathrm{~min}$ in $4 \%$ paraformaldehyde. The Aclar dish was then cut to form a round coverslip that was mounted tissue-side down on a drop of glycerol-based mounting medium containing Hoechst 33342.

Cultures to be stained for S100 were rinsed with L-15 before fixing in $4 \%$ paraformaldehyde in $0.1 \mathrm{M} \mathrm{PO}_{4}$ for $10 \mathrm{~min}$ at room temperature. The cultures were permeabilized with $4 \%$ paraformaldehyde plus $0.2 \%$ Triton $\mathrm{X}-100$ for $10 \mathrm{~min}$. The rabbit S100 polyclonal primary antibody (1:400; Dako, Carpinteria, CA), diluted with $0.1 \mathrm{M}$ phosphate buffer containing $10 \%$ HIGS and $0.2 \%$ Triton X-100, was applied for $60 \mathrm{~min}$ at room temperature. After incubation, the cultures were rinsed three times with $0.1 \mathrm{M} \mathrm{PO}_{4}$ containing $10 \%$ HIGS. The secondary antibody, goat anti-rabbit tetramethylrhodamine (ICN Cappel), was diluted 1:100 in 0.1 $\mathrm{M} \mathrm{PO}_{4} / 10 \% \mathrm{HIGS} / 0.2 \%$ Triton $\mathrm{X}-100$ and applied for $30 \mathrm{~min}$. Cultures were rinsed three times in $0.1 \mathrm{M} \mathrm{PO}_{4}$ containing $10 \%$ HIGS. Aclar dishes were cut and mounted as described above.

Cultures to be stained for MBP or neurofilaments were fixed in $4 \%$

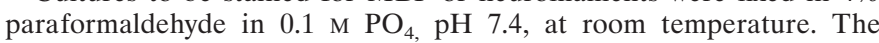
cultures were permeabilized for 15 min with $4 \%$ paraformaldehyde containing $0.2 \%$ Triton $\mathrm{X}-100$ in $0.1 \mathrm{M} \mathrm{PO}_{4}$ at room temperature. The cultures were then treated with ice-cold $50 \%$ acetone for $2 \mathrm{~min}$, ice-cold $100 \%$ acetone for $2 \mathrm{~min}$, and $50 \%$ acetone for $2 \mathrm{~min}$. Cultures were then rinsed twice with $0.1 \mathrm{M} \mathrm{PO}_{4}$ for 5 min each and incubated with mouse monoclonal anti-MBP (SMI 94; 1:1000; Sternberger Monoclonals, Inc., Lutherville, MD) or mouse monoclonal anti-neurofilament (SMI 31; 1:1000; Sternberger Monoclonals, Inc.) diluted in L-15 plus $10 \%$ HIGS for $30 \mathrm{~min}$ at room temperature. Rinsing three times in L-15 plus $10 \%$ HIGS was followed by goat anti-mouse fluorescein secondary antibody diluted 1:100 for 30 min. Cultures were rinsed three times with L-15 only and then once in $0.1 \mathrm{M} \mathrm{PO}_{4}$. Aclar dishes were trimmed as described above and mounted in Citifluor containing Hoechst 33342. 
Photomicrographs were taken on inverted Olympus (Tokyo, Japan) IX70 and Zeiss (Thornwood, NY) axioplan-2 microscopes.

Sudan black staining and counting. Cultures were fixed for at least 15 min in $4 \%$ paraformaldehyde, rinsed in $0.1 \mathrm{M} \mathrm{PO}_{4}$, and further fixed in $0.1 \% \mathrm{OsO}_{4}$ for $1 \mathrm{hr}$. The cultures were then rinsed three times in $0.1 \mathrm{M}$ $\mathrm{PO}_{4}$ followed by dehydration in 25, 50, and $70 \%$ ethanol, each for $5 \mathrm{~min}$. The $0.5 \%$ Sudan black $/ 70 \%$ ethanol solution was filtered before staining for $1 \mathrm{hr}$. The cultures were rehydrated in ethanol $(70 \%, 1 \mathrm{~min} ; 50 \%, 5$ min; $25 \%, 5 \mathrm{~min}$ ). The cultures were rinsed in $0.1 \mathrm{M} \mathrm{PO}_{4}$ and mounted on glycerin jelly after trimming the Aclar dish.

Myelinated axons were counted using a square grid eyepiece during three scans across the coverslip. The number of Sudan black-stained myelin sheaths crossing the scan line was counted. The first count was along a line at the center of the $25 \mathrm{~mm}$ dish; the second and third counts were done $7 \mathrm{~mm}$ above and below the center line. The numbers from the three scans were totaled. The mean myelin sheath count of these totals per culture type was obtained. In the experiments in serum-free medium, 12 cultures were analyzed in each experimental group, from four separate experiments. In the experiments with serum, six cultures were analyzed in each group in each of two separate experiments.

Statistics. For statistical analysis of Sudan black-stained cultures, data were analyzed using a one-factor ANOVA followed by the Bonferroni test between individual treatment groups. $p<0.001$ indicated a significant difference between groups.

Electron microscopy. Cultures were preserved in $2 \%$ phosphatebuffered glutaraldehyde (with $100 \mathrm{~mm}$ sucrose) overnight at $4^{\circ} \mathrm{C}$, followed by $2 \%$ buffered $\mathrm{OsO}_{4}$ for $1 \mathrm{hr}$ at room temperature. Cultures were further processed for EMbed plastic (Electron Microscopy Sciences, Fort Washington, PA) embedding. Areas were chosen for semithin sectioning; the $1 \mu \mathrm{m}$ sections were stained with toluidine blue/methylene blue/ sodium borate. Thin sections, stained with uranyl acetate and lead citrate, were examined in a Philips CM-10 electron microscope (FEI Company, Hillsboro, OR). Sections were cut perpendicular to the plane of the coverslip. Axonal diameter and ensheathment were measured in electron micrographs of SC/DRGN and EG/DRGN cultures, both grown in $\mathrm{NLA}+$ medium. Whether the axon was in contact with a glial process was noted; this contact was called ensheathment when at least $75 \%$ of the axolemma was covered by the glial process.

\section{RESULTS}

\section{SCs and EG were highly purified}

SC cultures were stained with p75 and S100 to ascertain purity. They contained 95-98\% p75+ cells (data not shown). EG cultures were double stained with S100/p75 or GFAP/p75 and counterstained with Hoechst 33342 to enable purity counts (Fig. 1). The EG preparations used in this study contained $90-95 \%$ p75+S100+ cells (Fig. $1 A-C$ ). Essentially all of the p75+ cells also strongly expressed GFAP (Fig. $1 D-F$ ). Purified EG preparations also contained a small percentage of GFAP + p $75-$ cells. These cells were probably astrocytes derived from olfactory bulb tissue that contaminated the nerve fiber layers.

\section{Purified control DRGN cultures sometimes contained SCs}

In each myelination experiment, control DRGN cultures, treated with FUDR to eliminate non-neuronal cells, were maintained to detect the presence of fibroblasts and SCs. A few remaining SCs would complicate the interpretation of results in DRGN cultures to which EG had been added. When the FUDR treatment succeeded, there were no non-neuronal cells remaining (Fig. 2A). None of the 12 control DRGN cultures fed NLA- medium for the entire 4 week period contained any myelin in Sudan blackstained preparations, but two of the cultures contained SCs and fibroblasts. In a different set of control DRGN cultures that were prepared for immunostaining for S100 and MBP, S100+ SCs were present in 3 of 12 cultures, but no $\mathrm{MBP}+$ myelin segments were detected (data not shown). The other cell type exhibited fibroblast-like morphology (data not shown). However, in 4 of 12 control DRGN cultures fed NLA- medium initially and then
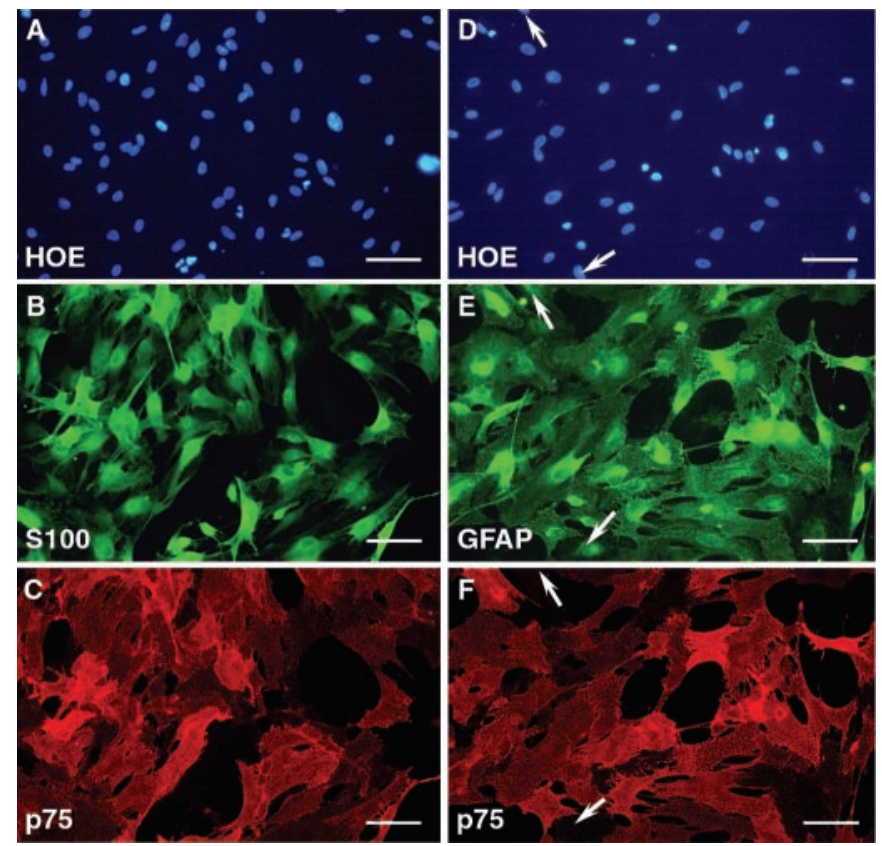

Figure 1. Immunopanned cells were highly enriched in p75+ EG. Comparisons of Hoechst $(H O E)$ staining $(A)$ with $\mathrm{S} 100(B)$ and p75 $(C)$ staining of the same field show that essentially all p75 + cells are also S100+. Similarly, a comparison of Hoechst staining $(D)$ with GFAP $(E)$ and p75 $(F)$ staining of the same field shows that essentially all p75+ cells are also GFAP + . Approximately $95 \%$ of the cells were p $75+$ at $3 \mathrm{~d}$ after immunopanning. A small number of p75-GFAP+ cells were found (arrows in $D-F$ ), suggesting that astrocytes were a contaminant of the preparation. This staining was performed after immunopanning with p75 antibody and maintenance for $3 \mathrm{~d}$ in vitro in DF-10S plus mitogens. Scale bars, $50 \mu \mathrm{m}$.

switched to NLA + medium, SCs, fibroblasts, and some myelin were observed (Fig. 2B); these four cultures contained one, three, one, and one SC-myelinated segment, respectively. The sizes of the neuronal somata appeared to be larger in cultures containing SCs than in those bereft of SCs. The other control DRGN cultures in this group contained only neurons.

\section{Myelin was formed in SC/DRGN cocultures without serum}

Twelve SC/DRGN cultures were fed NLA- medium (without serum) to initiate the proliferation of the added SCs. SCs proliferated and occupied the entire DRGN culture from the center to the ends of the axons. These cultures were maintained for an additional 2 weeks on NLA- medium to test whether myelin would form in the absence of ascorbate. The number of SCs was further increased, but Sudan black staining of all 12 cultures at 4 weeks on NLA - medium showed no myelin segments (Fig. 3A, $G$ ).

Twelve SC/DRGN cultures, given NLA+ medium (containing ascorbate but no serum) for the final 2 weeks, were also evaluated for myelin after Sudan black staining. In this group, numerous SCs were closely aligned along the axons. All 12 cultures contained myelin sheaths (Fig. 3C, $G$ ) (a mean count of $99.3 \pm 5.6$ sheaths/culture). This result was significantly different $(p<$ $0.001)$ from the control DRGN cultures $(0.5 \pm 0.26$ myelin segments).

\section{Myelin was rare in EG/DRGN cocultures without serum}

As with SC/DRGN cultures, one group of EG/DRGN cultures was kept in NLA- medium without serum for 4 weeks. EG proliferated in response to axons (the $24 \mathrm{hr}$ bromodeoxyuridine 


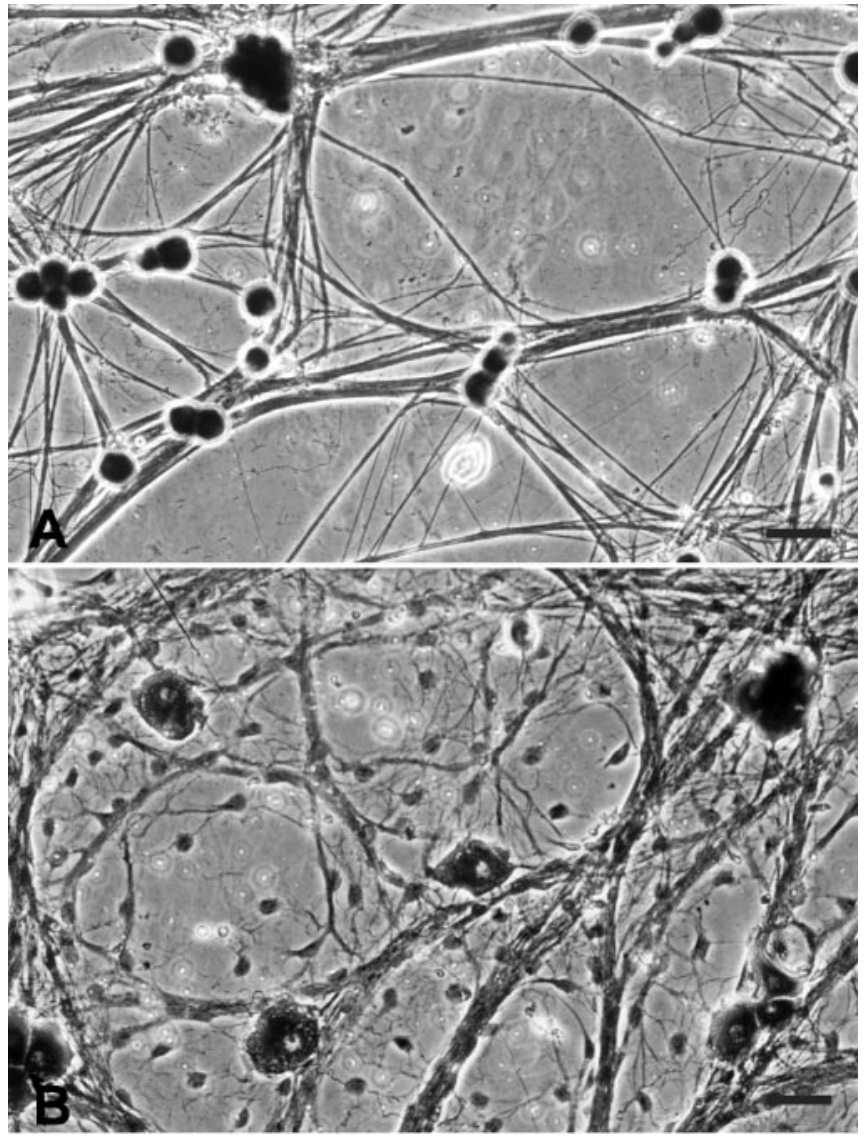

Figure 2. Purified control DRGN cultures occasionally contained SCs. Phase photomicrographs of two DRGN control cultures are shown. A, A culture maintained for 4 weeks in NLA- medium; in this culture, non-neuronal cells were successfully eliminated. $B$, A DRGN culture maintained in NLA+ medium for the final 2 weeks of the myelination period and containing a colony of cells morphologically similar to SCs. A few SC myelin sheaths were observed in 4 of 12 cultures in this group. Scale bars, $50 \mu \mathrm{m}$.

labeling index was $49 \%$, averaged over two separate experiments), and after 2 weeks EG were distributed throughout the entire culture. Whereas the initial rate of proliferation of the EG was comparable with that of SCs in SC/DRGN cocultures, the flat, broadly spread EG appeared to become confluent at a lower total cell density than did SCs (data not shown). EG/DRGN grown in NLA - medium for 4 weeks showed no myelin sheaths in any of the 12 cultures analyzed (Fig. 3B,G). EG/DRGN cultures maintained in NLA - for 2 weeks and in NLA+ myelinating medium for 2 weeks (Fig. 3D, G) contained myelin sheaths only rarely $(0.9 \pm 0.47)$ (Fig. $3 G)$. The number of myelin sheaths was not significantly different from that in control DRGN cultures fed NLA + medium $(0.5 \pm 0.26)$ (Fig. $3 G)$. The myelin segments that were counted were close to the culture center where the original drop of DRG cells was plated, in areas where surviving non-neuronal cells appeared occasionally in control DRGN cultures (data not shown). These results show that little, if any, myelin was formed compared with SCs in serum-free culture conditions.

\section{Myelin was not formed in EG/DRGN cocultures when serum was present}

To further test the ability of EG to produce myelin under conditions more like those in which EG myelin was reported previously
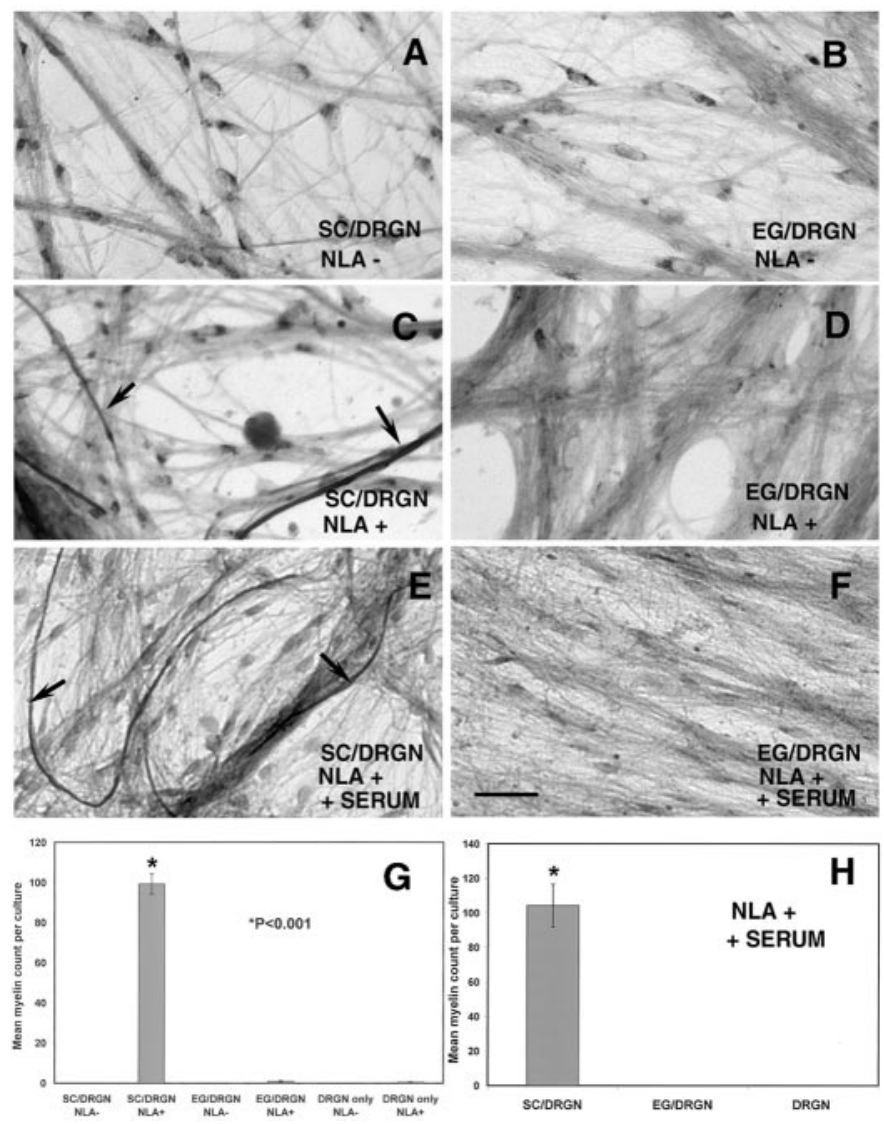

Figure 3. EG failed to form myelin under conditions that enabled SCs to produce myelin. $A, C$, Bright-field micrographs showing SC/DRGN cocultures in serum-free NLA medium without $(A)$ or with $(C)$ the addition of ascorbate. Myelin sheaths (arrows in $C$ ) are found only in cultures receiving ascorbic acid. EG/DRGN cultures are illustrated in $B$ (no ascorbate) and $D$ (with ascorbate); myelin was very rarely observed, and only in cultures with ascorbate. All cultures were fixed at the same time, at 2 weeks after the addition of serum and ascorbic acid. $E, F$, Bright-field micrographs showing cocultures in NLA medium supplemented with both serum and ascorbic acid. All cultures were fixed at the same time, at 3 weeks after the addition of serum and ascorbic acid. E, An SC/DRGN culture is illustrated; myelin (arrows) was readily observed in this culture group. $F$, An EG/DRGN culture exhibits no myelin, despite the presence of ascorbate. Scale bar, $25 \mu \mathrm{m}$. $G$, Graph comparing myelination in $\mathrm{SC} / \mathrm{DRGN}$ and EG/DRGN cultures under serum-free conditions. In the SC/DRGN group, a mean of $99 \pm 5.6$ myelin segments was counted per culture when fed ascorbate-containing medium. EG/DRGN cultures fed the same medium showed no or rare myelin segments, comparable with control DRGN cultures. Bar heights are the mean number of myelin segments counted per culture for each culture type $(n=12)$; error bar represents the SEM. * $p<0.001$ compared with control DRGN cultures. $H$, Graph comparing myelination in SC/DRGN and EG/DRGN cultures in the presence of serum; four cultures were counted in each group. Bar heights indicate the mean number of myelin segments counted per culture for each culture type $(n=4)$. Myelin was only observed in cultures to which SCs were added. A similar result was observed in a repeat experiment. *Error bar indicates SD.

(Devon and Doucette, 1992, 1995), SC/DRGN and EG/DRGN cocultures were prepared exactly as in the experiments just described, but with serum in the medium during the myelination period. After the addition of the SCs or EG, the cultures were maintained for a 2 week period in NLA - medium to allow the glia to proliferate. The cultures were then switched to NLA+ medium containing $10 \%$ heat-inactivated fetal bovine serum and kept for an additional 3 week period to allow myelination. In 

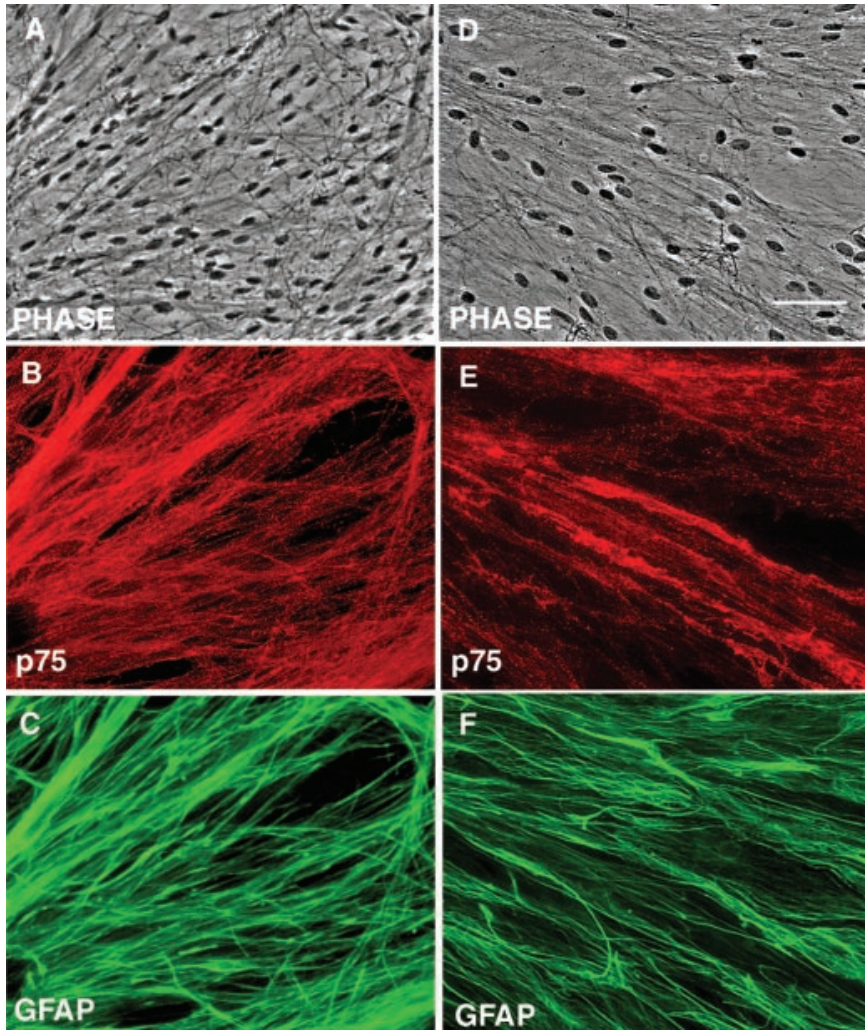

Figure 4. Most purified adult EG maintained a stable p75+GFAP+ phenotype during 5 weeks of coculture with DRGN. SC/DRGN and EG/DRGN cultures were fixed at 3 weeks after switching the cultures to myelination conditions (NLA medium supplemented with serum and ascorbic acid). $A-C$, A field in an SC/DRGN culture is illustrated. $A$, In this phase-contrast image, SC nuclei are observed in typical longitudinal arrays. $B, C$, Whereas individual cell boundaries are not easily observed at this low magnification, both p75 and GFAP staining were found where SCs were located, resulting in a high degree of correspondence between p75 and GFAP staining. $D-F$, A field in an EG/DRGN culture is illustrated. $D$, Nuclei of EG are visible in this phase-contrast image. The density of EG achieved after 5 weeks of coculture with neurons typically appears to be lower than that achieved by SCs. E, $F$, Despite the smaller number of EG, p75+GFAP+ processes cover much of the culture area. As with SCs, there is a strong correspondence between the position of EG nuclei and p75 and GFAP staining. Scale bar, $25 \mu \mathrm{m}$.

SC/DRGN cocultures, extensive myelin was formed during the final 2 weeks (Fig. 3E,H). No myelin was formed in EG/DRGN cocultures during the same period (Fig. $3 F, H$ ). The same result was obtained in a repeat experiment. In both experiments in serum-containing medium, myelin was not formed in control DRGN cultures to which no exogenous glia were added.

\section{Typical SC-like ensheathment was lacking in EG/DRGN cultures}

A defining property of SCs is their unique affinity for axons. The manner in which SCs associated with axons was an easily recognizable feature of SC/DRGN cultures and was notably different from the way that EG associated with axons in EG/DRGN cultures. In the present study, subtle differences were observed by light microscopy, and more profound differences were observed by electron microscopy. In SC/DRGN cultures observed by light microscopy, the SCs were arranged in linear arrays that appeared to follow the course of underlying axon bundles (Fig. 4A). In contrast, EG appeared much flatter, interacting with axons primarily by extending flattened, nearly invisible processes between fascicles of axons. Although this can be seen with the microscope, it is not clear in photographs (Fig. 4D). Recent confocal analysis by another investigator in our laboratory has shown that axon bundles defasciculate and that fine axons fan out profusely across the EG surface (I. Wanner, unpublished observation). Interestingly, at the time the cultures were terminated for analysis, most SCs (Fig. 4B,C) and EG (Fig. 4E,F) associated with axons continued to strongly express both p75 and GFAP. Because it is well known that p75 and GFAP are not expressed in myelinating $\mathrm{SCs}$, it is likely that it is the nonmyelinating SCs in SC/DRGN cocultures that are positive for these markers.

Cultures were studied by electron microscopy to better characterize axon-glial relationships in SC/DRGN and EG/DRGN cultures. There was a marked difference in ensheathment. In $\mathrm{SC} / \mathrm{DRGN}$ cultures, in addition to myelin, many axons (smaller in diameter than myelinated axons but not of the smallest diameter) were individually encircled by SC cytoplasm in ascorbatecontaining medium (Fig. 5, arrow). In contrast, thin EG processes were often adjacent to axons but did not completely encircle them (Fig. 6). Occasionally, EG processes were observed to partially engulf axons; when this engulfment covered $75 \%$ of the axonal surface the axons were considered to be ensheathed (Table 1). Very different from SCs, EG extended long, thin meandering sheets that partitioned fascicles of axons (Fig. 6A) and that sometimes spanned the entire thickness of the culture. Moreover, far fewer axons were contacted by EG than by SCs (Table 1). The relationship of EG and axons just described was confirmed in thin sections cut parallel to the coverslip, where much more tissue was available for examination (data not shown).

The morphological appearance of EG/DRGN cultures was similar in ascorbate-lacking and ascorbate-containing conditions and regardless of whether serum was present. The observed differences between SC/DRGN and EG/DRGN cultures were not caused by differences in the density or number of the axons, because at the time SCs and EG were added to the cultures, these parameters would have been identical in all cultures. The differences must have arisen subsequent to the addition of glia.

It was mentioned above that occasionally a few myelin sheaths were found in EG/DRGN cultures fed ascorbate-containing serum-free medium. It was not possible to determine conclusively, on the basis of fine structure, whether the myelin had been formed by EG or SCs that had survived the FUDR treatment. Control DRGN cultures were studied by electron microscopy to determine the appearance of surviving SCs that formed myelin under the conditions of these experiments, in hopes of being able to distinguish between SC and EG cytoplasm. Ultrastructural differences between the cytoplasm of myelinating SCs and the rare myelinating cells in EG/DRGN cultures were not apparent.

\section{DISCUSSION}

In the foregoing comparison of the abilities of adult-derived SCs and adult-derived EG to produce myelin sheaths when presented with DRGN axons in vitro, the number of myelin sheaths produced in SC/DRGN cultures was far higher than in EG/DRGN cultures. The number of sheaths produced in EG/DRGN cultures was not significantly different from the number of sheaths produced in control DRGN cultures that did not receive additional cells. The latter result demonstrates that myelination by SCs remaining in DRGN cultures after the antimitotic treatment sometimes occurs and suggests that some or all of the myelin produced in EG/DRGN cultures could have been produced by these residual SCs. Most importantly, striking differences in the 
Figure 5. Myelin formation and ensheathment in an SC/DRGN culture. In an SC/ DRGN culture fed ascorbate-containing but serum-free medium, a myelin sheath formed by an SC is illustrated in the center. Surrounding it are nonmyelinated, ensheathed axons; complete encirclement of an axon by $\mathrm{SC}$ cytoplasm is indicated by the arrow. Scale bar, $1 \mu \mathrm{m}$.

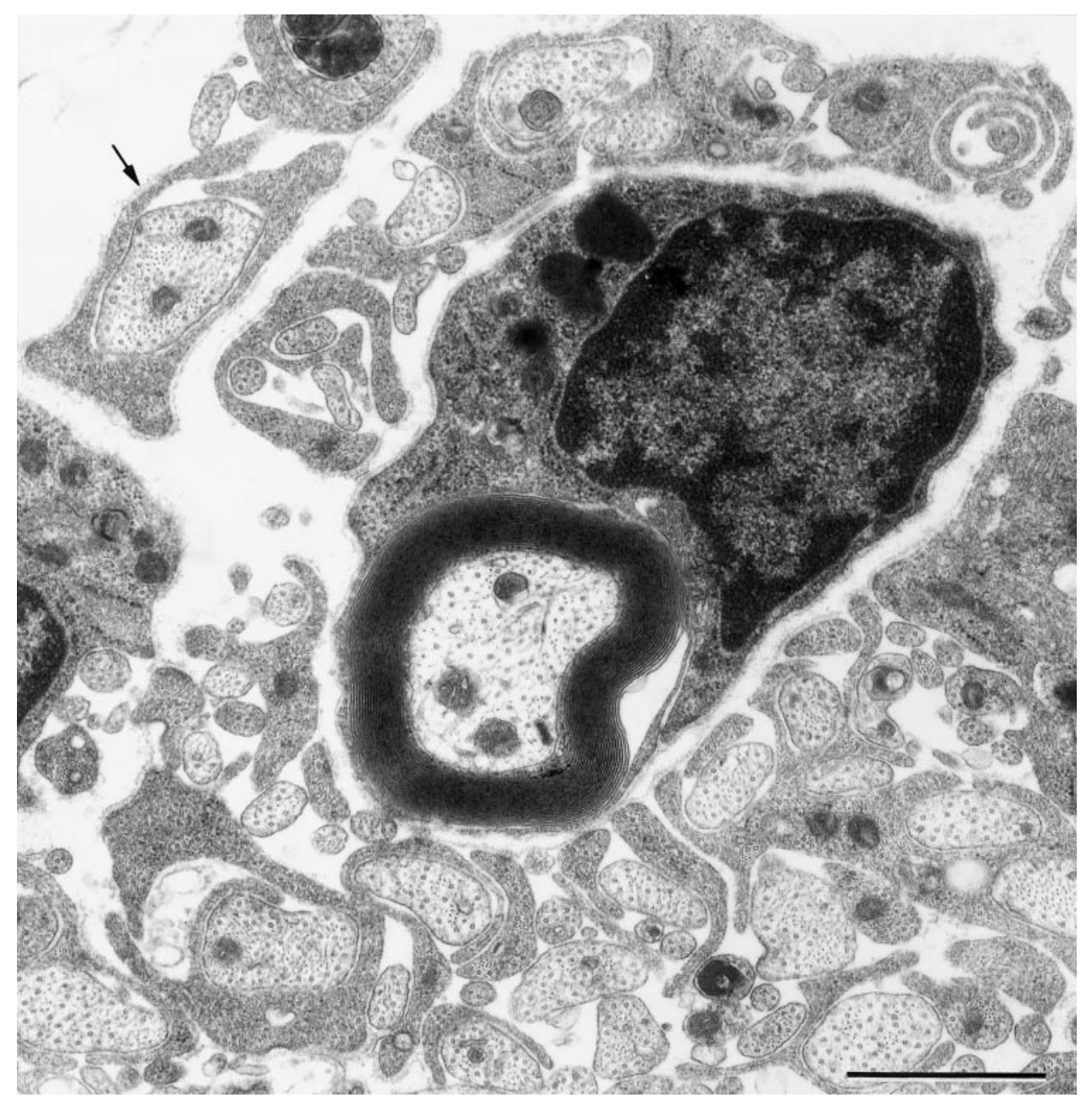

conformation of cellular processes and relationship to axons were observed between SC/DRGN and EG/DRGN cultures. Whereas the SCs displayed relatively short, thick processes that were observed to engulf individual or small bundles of axons and to segregate larger axons into one-to-one relationships, the EG displayed large flattened sheets that partitioned fascicles of axons, sometimes spanning the thickness of the culture. SCs exhibited behavior typical of SCs in peripheral nerves, whereas EG exhibited behavior resembling, in many respects, that of EG in the olfactory system (Raisman, 1985). Thus, despite an extended period in culture with DRGN, the EG did not exhibit an SC-like configuration.

These tissue culture results do not support the conclusion reached in other reported studies of the ability of EG to produce myelin. SC-like myelination was observed in cultures of DRGN to which EG were added (Devon and Doucette, 1992, 1995) and when EG were transplanted into a variety of lesions in the spinal cord of adult rats (Franklin et al., 1996; Li et al., 1997, 1998; Imaizumi et al., 1998, 2000; Barnett et al., 2000; Kato et al., 2000). In these previous studies, myelination was observed when the EG preparation was derived from developing or adult tissues and when the preparation was used without purification or with purification by immunoselection with specific antibodies such as p75. On the strength of these observations, the view that EG can produce myelin has become firmly established and the use of transplanted EG as a therapeutic strategy to repair myelin in demyelinating disease has been proposed (for review, see Franklin and Barnett, 2000).

It has been reported that there are two different types or phenotypes of EG in cultures derived from olfactory nerves and bulb (Pixley, 1992; Franceschini and Barnett, 1996; Ramón-Cueto and Avila, 1998); one of these is p75 positive and the other is p75 negative. The EG suspensions used in the present study were purified using immunopanning to select p75-expressing cells. Our results suggest that, under the conditions tested (which promote myelination), p75-expressing EG do not have the capacity to produce myelin. One explanation for this could be that the precursors of hypothetical myelinating EG are p75 negative and therefore not selected by the immunopanning. The failure of p75-positive EG to produce myelinating EG would then constitute a major difference between EG and SCs, because the precursors of myelinating SCs are p75 positive (Jessen et al., 1994). The absence of SC myelin in the EG/DRGN cultures also indicates that p75 immunopanning of adult-derived olfactory bulb glia does not yield a significant number of SCs as contaminants in the purified EG preparation.

It is possible that EG could form myelin under certain conditions. Although myelination by EG is not normally observed within the olfactory nerves or in the nerve fiber layer of the olfactory bulb, this lack of myelin might reflect the specific properties of the resident olfactory axons. It is noteworthy, however, that in all the reports suggesting that EG can myelinate axons, the 

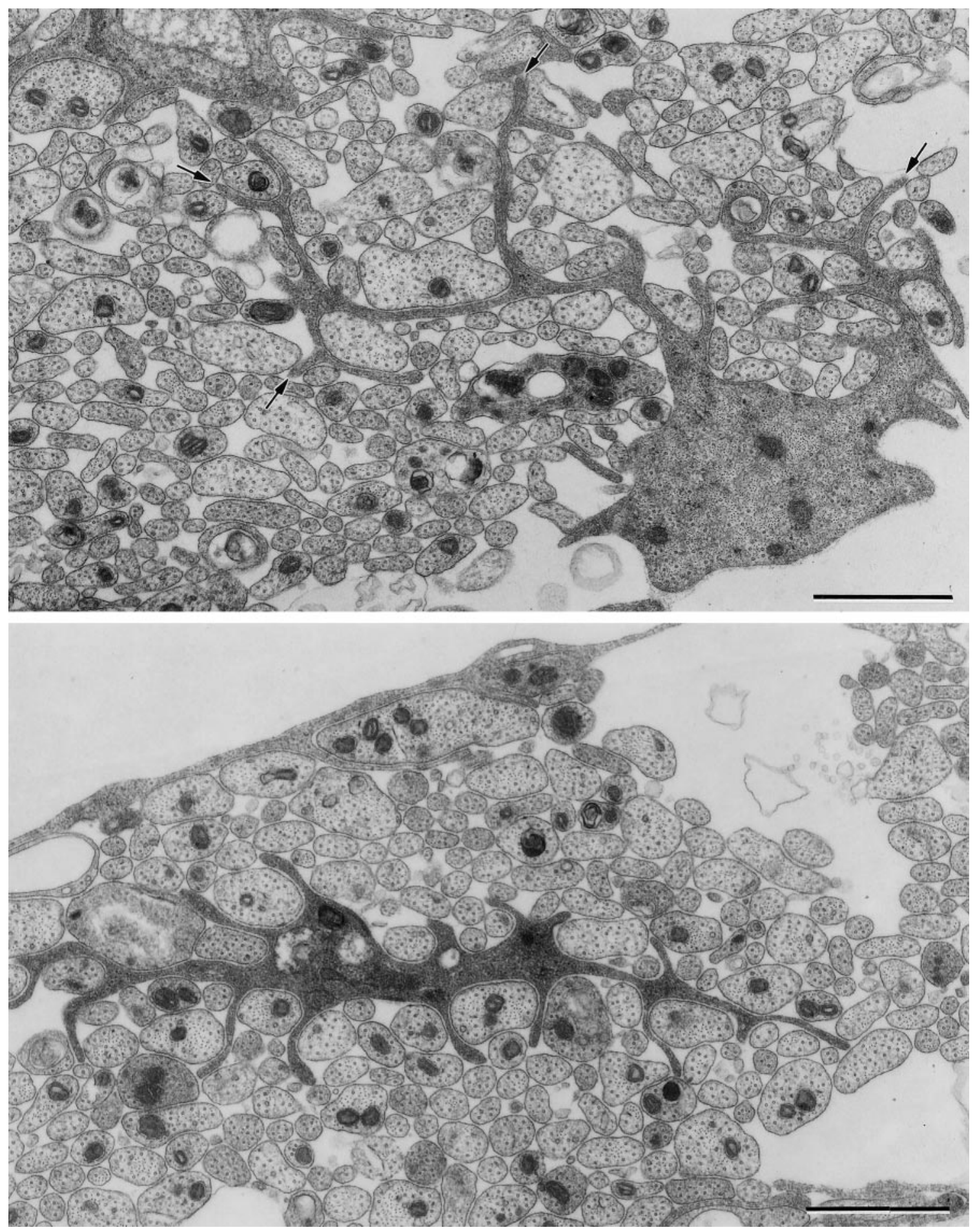

Figure 6. Lack of typical SC-type ensheathment in an EG/DRGN culture. These two panels illustrate two examples of the flat meandering EG processes (arrows, top panel) that only partially encircle axons or partition fascicles of axons but do not provide furrows for each axon as seen in SC/DRGN cultures. The EG cytoplasm contains numerous intermediate filaments and microtubules (best seen in the cell in the top panel). The culture was maintained in ascorbate-containing, serum-free NLA medium. Scale bar, $1 \mu \mathrm{m}$.

myelin produced was morphologically identical to the myelin produced by SCs. That myelinating EG would adopt an SC shape and produce myelin identical to SC myelin is surprising and puzzling in view of the different embryonic origins of SCs and EG (Ramón-Cueto and Avila, 1998), the differences in the conformation of SCs and EG in their respective in situ locations, and, most importantly, the differences in the manner in which the processes of EG and of SCs relate to the axons they ensheathe. SCs in nerve fascicles in vivo and in culture with neurons exhibit a torpedo shape with a cytoplasm-rich perikaryon and processes that engulf axons within surface furrows and form mesaxons. In contrast, EG in vivo are dramatically polarized cells, expanding their basal surface into broad diaphanous processes that contact similar processes of other EG to form a continuous sheath that surrounds 


\begin{tabular}{|c|c|c|c|c|}
\hline & No glial contact & Glial contact & Ensheathment & Myelin \\
\hline \multicolumn{5}{|c|}{ Number of axons counted ( $\%$ total axons) } \\
\hline $\mathrm{SCs}^{a}$ & $66(7.4)$ & $605(72.3)$ & $162(19.5)$ & $8(0.8)$ \\
\hline $\mathrm{EG}^{b}$ & $526(62.6)$ & $302(35.9)$ & $11(1.3)$ & $1(0.1)$ \\
\hline \multicolumn{5}{|c|}{ Mean axonal diameter $(\mu \mathrm{m}) \pm \mathrm{SD}$} \\
\hline $\mathrm{SCs}$ & $0.17 \pm 0.06$ & $0.21 \pm 0.09$ & $0.32 \pm 0.13$ & $0.78 \pm 0.21$ \\
\hline EG & $0.19 \pm 0.06$ & $0.20 \pm 0.10$ & $0.30 \pm 0.10$ & 1.48 \\
\hline
\end{tabular}

Axons were considered to be ensheathed when at least $75 \%$ of the axolemma was covered by a glial process.

${ }^{a}$ A total of 841 axons was counted throughout five electron micrographs of SC/DRGN cultures at $8900 \times$ magnification.

${ }^{b} \mathrm{~A}$ total of 840 axons was counted throughout two electron micrographs of EG/DRGN cultures at $8900 \times$ magnification.

olfactory axon bundles (Raisman, 1985). The apical surface of individual EG is extended into the interior of the axon bundle as multiple tentacle-like processes that further partition and organize the bundle. EG do not engulf axons within surface furrows or grooves. Cells exhibiting this jellyfish-like morphology were not illustrated or described as present in previous reports of EG myelination. However, in one of the studies, thin processes that could be those of EG were closely applied to the axons ( $\mathrm{Li}$ et al., 1998).

In testing the hypothesis that EG are myelination-competent, a number of precautionary measures need to be taken to ensure that results are interpreted without error. Perhaps foremost of these is to ensure that the EG preparations used in such studies do not contain SCs. In experiments in our laboratory over many years using dissociated, meninges- and root-free spinal cord as a source of CNS glia, SCs have been a consistent contaminant of the resulting cultures (Wood, unpublished observations; Blakemore et al., 1987). Possibly this is because SCs are normally present within CNS tissue in association with sympathetic axons that innervate smooth muscle in the walls of larger-diameter blood vessels. When olfactory bulb tissue is dissociated, such SCs might be obtained as contaminants of the EG suspension. Because SCs share many antigenic markers with EG, the two cell types cannot be separated easily by any of the approaches that are commonly used for EG purification, such as immunoselection with p75 or O4 antibodies. The proliferation of EG and SCs is driven by the same mitogenic factors (Porter et al., 1986; Yan et al., 2001). The proliferation and differentiation of even a few SCs in the EG preparations, subsequent to transplantation to either cultures or animals, could explain the presence of SC-like myelin.

A second potential pitfall in these studies is the migration of host SCs into CNS lesions that receive EG transplants. SC immigration occurs rapidly and spontaneously after many types of spinal cord injury (Bunge et al., 1994; Brook et al., 1998). In addition, a rapid angiogenic response is induced by the injection of EG and other cell types into lesioned corticospinal tract ( $\mathrm{Li}$ et al., 1998). The ingrowth of new vessels clothed in basal lamina might promote the migration of SCs into the lesion. This possibility may well apply to the $\mathrm{x}$-irradiation/ethidium bromide model of persistent demyelination that was used in several studies. Although the angiogenic response to the placement of EG in these demyelinated lesions has not been determined, such a response seems likely. In addition, because EG have the unique property of extensive migration into host tissue containing astrocytes, the presence of EG in the tissue adjacent to the lesion might enable the migration of host SCs from the adjacent tissue into the lesion, although such migration does not occur without the EG implant (Franklin et al., 1996; Barnett et al., 2000). Only when EG were injected into the adult rat spinal cord next to an $\mathrm{SC}$ bridge were axons that had regenerated into the bridge able to extend from the bridge into the cord (Ramón-Cueto et al., 1998). Although the mechanism by which EG foster the regeneration of axons from the $\mathrm{SC}$ bridge is not known, it is reasonable to suggest that EG change the cord milieu in such a way as to enable SC migration into the cord, which in turn contributes to axonal regeneration from the bridge.

Because these potential pitfalls were not ruled out in the publications cited above reporting EG myelination in vivo, it is our view that there is some question as to whether EG have the potential to produce myelin. Additional studies, possibly involving the use of purified, genetically labeled EG, are needed to fully resolve this issue. Although human EG have been transplanted to demyelinated lesions in rat spinal cord induced by $\mathrm{x}$-irradiation and ethidium bromide injection, and although the presence of human cells has been verified in the lesion after remyelination (Kato et al., 2000), the relationship between myelin and the human cells remains to be shown.

\section{REFERENCES}

Barnett SC, Alexander CL, Iwashita Y, Gilson JM, Crowther J, Clark L, Dunn LT, Papanastassiou V, Kennedy PGE, Franklin RJM (2000) Identification of a human olfactory ensheathing cell that can effect transplant-mediated remyelination of demyelinated CNS axons. Brain 123:1581-1588.

Blakemore WF, Crang AJ, Evans RJ, Patterson RC (1987) Rat Schwann cell myelination of de-myelinated cat axons: evidence that injections of cell suspensions of CNS tissue result in Schwann cell remyelination. Neurosci Lett 77:15-19.

Brook GA, Plate D, Franzen R, Martin D, Moonen G, Schoenen J, Schmitt AB, Noth J, Nacimiento W (1998) Spontaneous longitudinally oriented axonal regeneration is associated with the Schwann cell framework within the lesion site following spinal cord compression injury of the rat. J Neurosci Res 54:51-65.

Bunge MB, Holets VR, Bates ML, Clarke TS, Watson BD (1994) Characterization of photochemically induced spinal cord injury in the rat by light and electron microscopy. Exp Neurol 127:76-93.

Casella GTB, Bunge RP, Wood PM (1996) Improved method for harvesting human Schwann cells from mature peripheral nerve and expansion in vitro. Glia 17:327-338.

Devon R, Doucette R (1992) Olfactory ensheathing cells myelinate dorsal root ganglion neurites. Brain Res 589:175-179.

Devon R, Doucette R (1995) Olfactory ensheathing cells do not require L-ascorbic acid in vitro to assemble a basal lamina or to myelinate dorsal root ganglion neurites. Brain Res 688:223-229.

Franceschini IA, Barnett SC (1996) Low-affinity NGF-receptor and E-N-CAM expression define two types of olfactory nerve ensheathing cells that share a common lineage. Dev Biol 173:327-343.

Franklin RJ, Barnett SC (1997) Do olfactory glia have advantages over Schwann cells for CNS repair? J Neurosci Res 50:665-672.

Franklin RJ, Barnett SC (2000) Olfactory ensheathing cells and CNS regeneration: the sweet smell of success? Neuron 28:15-18.

Franklin RJ, Gilson JM, Franceschini IA, Barnett SC (1996) Schwann cell-like myelination following transplantation of an olfactory bulbensheathing cell line into areas of demyelination in the adult CNS. Glia 17:217-224.

Imaizumi T, Lankford KL, Waxman SG, Greer CA, Kocsis JD (1998) 
Transplanted olfactory ensheathing cells remyelinate and enhance axonal conduction in the demyelinated dorsal columns of the rat spinal cord. J Neurosci 18:6176-6185.

Imaizumi T, Lankford KL, Kocsis JD (2000) Transplantation of olfactory ensheathing cells or Schwann cells restores rapid and secure conduction across the transection spinal cord. Brain Res 854:70-78.

Jessen KR, Brennan A, Morgan L, Mirsky R, Kent A, Hashimoto Y, Gavrilovic J (1994) The Schwann cell precursor and its fate: a study of cell death and differentiation during gliogenesis in rat embryonic nerves. Neuron 12:509-527.

Kato T, Honmou O, Uede T, Hashi K, Kocsis J (2000) Transplantation of human olfactory ensheathing cells elicits remyelination of demyelinated rat spinal cord. Glia 30:209-218.

Kleitman N, Wood PM, Bunge RP (1998) Tissue culture methods for the study of myelination. In: Culturing nerve cells, Ed 2 (Banker GA, Goslin K, eds), pp 545-594. Boston: MIT.

Li Y, Field PM, Raisman G (1997) Repair of adult rat corticospinal tract by transplants of olfactory ensheathing transplants. Science 277:2000-2002.

Li Y, Field PM, Raisman G (1998) Regeneration of adult rat corticospinal tract axons induced by transplanted olfactory ensheathing cells. J Neurosci 18:10514-10524.

Morrissey TK, Kleitman N, Bunge RP (1991) Isolation and functional characterization of Schwann cells derived from adult peripheral nerve. J Neurosci 11:2433-2442.

Pixley SK (1992) The olfactory nerve contains two populations of glia, identified both in vivo and in vitro. Glia 5:269-284.

Plant GW, Ramón-Cueto A, Bunge MB (2001) Transplantation of Schwann cells and ensheathing glia to improve regeneration in adult spinal cord. In: Axonal regeneration in the central nervous system (Ingoglia NA, Murray M, eds), pp 529-561. New York: Marcel Dekker.
Porter S, Clark MB, Glaser L, Bunge RP (1986) Schwann cells stimulated to proliferate in the absence of neurons retain full functional capability. J Neurosci 6:3070-3078.

Raisman G (1985) Specialized neuroglial arrangement may explain the capacity of vomeronasal axons to reinervate central neurons. Neuroscience 14:237-254.

Ramón-Cueto A, Avila J (1998) Olfactory ensheathing glia: properties and function. Brain Res Bull 46:175-187.

Ramón-Cueto A, Nieto-Sampedro M (1992) Glial cells from adult rat olfactory bulb: immunocytochemical properties of pure cultures of ensheathing cells. Neuroscience 47:213-220.

Ramón-Cueto A, Nieto-Sampedro M (1994) Regeneration into the spinal cord of transected adult rat spinal cord is promoted by ensheathing glia transplants. Exp Neurol 127:232-244.

Ramón-Cueto A, Perez J, Nieto-Sampedro M (1993) In vitro enfolding of olfactory neurites by p75 receptor positive ensheathing cells from adult rat olfactory bulbs. Eur J Neurosci 5:1172-1180.

Ramón-Cueto A, Plant GW, Avila J, Bunge MB (1998) Long-distance axonal regeneration in the transected adult rat spinal cord is promoted by olfactory ensheathing glia transplants. J Neurosci 18:3803-3815.

Ramón-Cueto A, Cordero MI, Santos-Benito FF, Avila J (2000) Functional recovery of paraplegic rats and motor axon regeneration in their spinal cords by olfactory ensheathing cells. Neuron 25:425-435.

Xu XM, Chen A, Guénard V, Kleitman N, Bunge MB (1997) Bridging Schwann cell transplants promotes axonal regeneration from both the rostral and caudal stumps of transected adult rat spinal cord. J Neurocytol 26:1-16.

Yan H, Bunge MB, Wood PM, Plant GW (2001) Mitogenic response of adult rat olfactory ensheathing glia to four growth factors. Glia 33:334-342. 